\title{
Simulation modelling of a patient surge in an emergency department under disaster conditions
}

\author{
Muhammet Gul ${ }^{1, *}$ and Ali Fuat Guneri ${ }^{1}$ \\ ${ }^{1}$ Department of Industrial Engineering, Yildiz Technical University \\ 34349, Besiktas-Istanbul, Turkey \\ E-mail: 〈\{mgul, guneri\}@yildiz.edu.tr $\rangle$
}

\begin{abstract}
The efficiency of emergency departments (EDs) in handling patient surges during disaster times using the available resources is very important. Many EDs require additional resources to overcome the bottlenecks in emergency systems. The assumption is that EDs consider the option of temporary staff dispatching, among other options, in order to respond to an increased demand or even the hiring temporarily non-hospital medical staff. Discrete event simulation (DES), a well-known simulation method and based on the idea of process modeling, is used for establishing ED operations and management related models. In this study, a DES model is developed to investigate and analyze an ED under normal conditions and an ED in a disaster scenario which takes into consideration an increased influx of disaster victims-patients. This will allow early preparedness of emergency departments in terms of physical and human resources. The studied ED is located in an earthquake zone in Istanbul. The report on Istanbul's disaster preparedness presented by the Japan International Cooperation Agency (JICA) and Istanbul Metropolitan Municipality (IMM), asserts that the district where the ED is located is estimated to have the highest injury rate. Based on real case study information, the study aims to suggest a model on pre-planning of ED resources for disasters. The results indicate that in times of a possible disaster, when the percentage of red patient arrivals exceeds $20 \%$ of total patient arrivals, the number of red area nurses and the available space for red area patients will be insufficient for the department to operate effectively. A methodological improvement presented a different distribution function that was tested for service time of the treatment areas. The conclusion is that the Weibull distribution function used in service process of injection room fits the model better than the Gamma distribution function.
\end{abstract}

Key words: emergency departments, patient surge, disaster time, simulation

Received: November 10, 2014; accepted: October 11, 2015; available online: October 31, 2015

DOI: 10.17535 /crorr.2015.0033

\section{Introduction}

In recent years, Turkey has faced various disasters. The consequences of the Marmara (17th August 1999) and Düzce (23rd November 1999) earthquakes,

\footnotetext{
${ }^{*}$ Corresponding author.
} 
according to the official figures, were that 18,287 people lost their lives, 46,857 were injured, 164,711 workplaces or houses were moderately damaged and 1,100,000 people became homeless [1]. On 23rd October 2011, an earthquake with a magnitude of 7.2 on the Richter scale occurred in Van and 604 people lost their lives [7]. More recently, 301 miners died due to the carbon monoxide poisoning and 140 injured in an underground coalmine in Soma, Manisa. Disaster events similar to these cause an increase the influx of emergency patients to hospital EDs. Therefore, effective and functional utilization of ED resources to meet patient surge using appropriate methods is very crucial in such circumstances.

Simulation of modeling is extensively applied to ED operations in available literature. The studies focus on wait time reduction, patient productivity, staff allocation, facility redesign, and cost control. Numerous literature surveys address simulations in EDs. Lim et al. [21] investigated twenty-nine studies to evaluate hospital ED waiting-time reduction strategies using mathematical simulation techniques for queuing models, DES, system dynamics (SD) and agent-based simulation (ABS). Jun et al. [15] combined DES applications in health care clinics and clinic systems (hospitals, EDs, outpatient clinics and pharmacies). In recent literature, studies by Choon et al. [6] and Konrad et al. [19] have focused on decreasing total patient stay in EDs. Morgareidge et al. [22] integrated DES and space syntax analysis (SAA) to optimize patient flow and to design the premises of an ED. In another study, Kang et al. [17] revealed the impact of various patient admission processes on patient flow in an ED. A miscellaneous example on ED simulation was presented by Kadri et al. [16]. They developed a simulation-based DSS to prevent and predict strain situations in an ED with the aim of improving management by the hospital system. In regards to patient productivity, Al-Refaie et al. [3] proposed a DES model to reduce average patient waiting time, to improve utilization of nursing staff and increase the number of treated patients.

In addition to all these recent studies, simulation of modeling is a significant analytical tool in analyzing the behavior of ED systems under disaster conditions where the aim is to improve preparedness. To reduce the impact of disasters, ED executives should improve strategies to determine preparedness in terms of resource efficiency, system performance and the ability to eliminate bottlenecks with the aid of scenarios thoroughly tested using constructed simulation models $[7,11,12]$. The available literature provides limited studies, such as [25], [5], [14], [2] and [23]. Xiao et al. [25] optimized workflow during a patient surge of a disaster event using an ED DES model. Joshi and Rys [14] and Patvivatsiri [23] studied terror disaster cases. The first paper analyzes different arrival patterns in terms of ED capacity during a conventional terror disaster event using DES. In the second paper, patient flow is analyzed throughout the treatment process, utilization of ED resources are assessed, the impact of a hypothetical bioterrorist attack is evaluated, and the 
appropriate resource and staff levels for such a bioterrorism scenario is determined. Al-Kattan and Abboud [2] modeled ED operations during disaster times as well normal times using a different scenario.

These studies mentioned above have limitations. Xiao et al. [25] and AlKattan and Abboud [2] use only patient waiting time as a performance measure. In this study, multiple key performance indicators (KPIs) including length of stay (LOS), utilization of medical staff and, utilization of locations are considered. Unlike the current study, Joshi and Rys [14] and Patvivatsiri [23] focus on terror disaster events. This study relies on patient arrival percentages from the study of a previous catastrophic earthquake event in Turkey. Therefore, the topic in question is directly related to an earthquake disaster event.

The inference can be made that ED simulation must focus on conditions caused by a disaster event and early preparedness of emergency departments in terms of physical and human resources. An increasing complexity of ED processes compared to the normal times leads to seeking ways to normalize and then enhance the system. Here, simulation modelling assumes an important role for the functioning of EDs. This study investigates both normal time analysis of an ED and a scenario disaster scenario, and considers the increased patient arrivals of disaster victims in a public hospital located in Istanbul's earthquake zone.

In summary this study makes the following contributions to existing literature: (1) It presents a DES model of an ED that enables generating various scenarios depending on patient arrival variability created by benefiting from experienced gained in a previous earthquake. The model generates outputs for several KPIs that have not been taken into consideration by previous studies. (2) It runs and validates the model by changing the distribution function for treatment time service. A statistical difference between models with different distribution functions has been observed. (3) It presents a real-world case study of an ED in a public hospital in Istanbul, Turkey. The simulation model performed in this ED is the first attempt at answering the question "Is the ED ready to meet a patient surge after a major earthquake, which according to the authorities is expected to be unavoidable in the Istanbul province".

This study aims to show the impacts of various arrival configurations on ED performance indicators such as LOS, utilization of human and location resources with a fixed number of staff. Moreover, the threshold value $(20 \%$ in this study) where existing resources may be insufficient for an ED was also determined. Therefore, our endeavor was to suggest a strategy for the functioning of an ED in the event of a disaster.

The paper has been organized in the following manner. Section 2 presents a DES methodology and the observed ED environment. The simulation model is presented in Section 3. Then, Section 4 presents the results of both a normal 
operating and disaster scenario. The last section provides a conclusion and the constraints of the study, with future recommendations highlighted.

\section{Method of discrete event simulation}

Systems that have dynamic behavior are hard to describe using static dependencies between variables. To analyze such systems, using simulation modelling is more appropriate [4]. It has penetrated several areas of use from manufacturing to healthcare. Numerous authors have applied simulation for problems associated with healthcare facilities such as EDs, inpatient units, intensive care units and outpatient clinics. The reason for choosing simulation modeling for other research methods stems from the stochastic nature of patient arrival and treatment processes [10]. Literature indicates the exceptional difficultly with modeling the complexity of EDs using a single analytical model [8]. Therefore, simulation models are required for monitoring ED systems.

Discrete-event simulation is one of the best-known simulation methods used for building healthcare facility models. It is based on the idea that the modeler views the system to be modeled as a process [4]. DES modeling is widely applied to modeling ED operations [9]. ED operations include delays, services offered by various medical resources, choosing the process branch, and other factors. Since an ED DES model is based on tracking patients as entities, it has a queuing structure [13]. The reason for choosing DES modeling among other simulation methodologies such as ABS and SD modeling stems from the followings: (1) DES can easily model stochastic factors affecting ED system; (2) EDs have queues and performance based on waiting times. Therefore, the queuing structure can be modeled by DES easily. (3) DES presents visual representation by its animation feature. It provides ED executive to monitor their systems easily. (4) Individual patient behavior at the EDs can be monitored using DES modeling.

\section{Simulation of the case study department}

\subsection{Emergency department environment}

The observed emergency department treats approximately 800-1000 patients a month in Istanbul. It implements a three color-triage system: green, yellow and red. Green stands for patients who have the least severe cases and red represents real life-threatening emergencies. The total value of current bed capacity in the red, yellow and green categories, as well as in child observation and the injection area is 24 . The department also has a laboratory for blood tests, an X-ray room as well as a computer-aided tomography (CT), an 
ultrasonography (USG) and a plaster room. It employs 2 physicians, 4 practitioners, 30 nurses, 6 registrars and a few technicians. All of the staff including the medical and auxiliary personnel provides services to patients arriving $7 / 24$ and 52/365. They often work in two shifts. The shift hours are from 8 am to $4 \mathrm{pm}$ for physicians and nurses, whereas technicians work in single 24-hour shifts.

\subsection{Description of process and data collection}

Arrivals at the ED happen in one of two ways: walk-in or ambulance. Since ambulance patients have a life-threatening condition, they bypass the registration process and enter directly from the front door of the ED to the red patient area (resuscitation). Walk-in patients are registered and triaged by a registrar and then a nurse. After registration, they are sent to the green patient area or injection area. Patients who are sent to the green area are assigned to an available bed, if the treatment is deemed to take a short time or are sent to the yellow patient area (used as a patient observation room), if treatment is deemed to last a longer period. Moreover, transfers from the green and yellow patient area to the red area are performed if needed (see the patient flow in the ED in Figure 1).

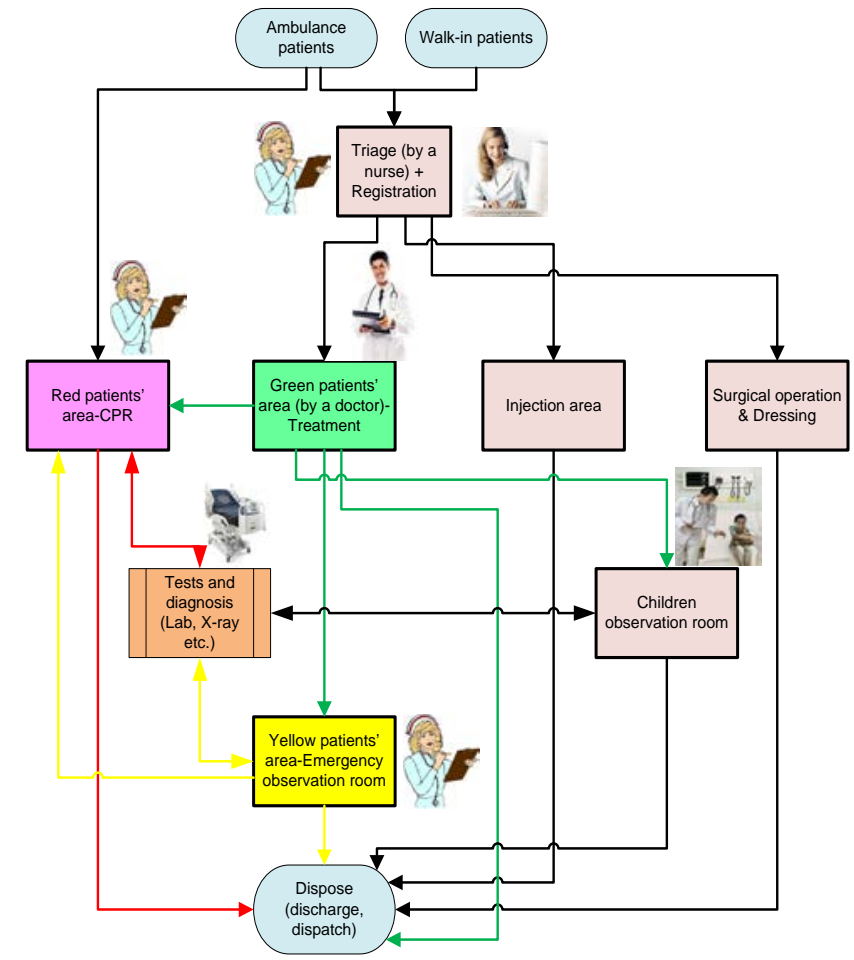

Figure 1: Patient flow in the ED in the observed hospital 
Data collection and analysis is a crucial phase in simulation projects. Reliability of the simulation model depends on the quality of input data and assumptions [2]. Therefore, in this study, historical data obtained from the hospital information management system (called the $H B Y S$ ) database was used. The data consisted of 266,693 patient records from the ED between 1st January and 31st December 2012. The data set includes the treatment area to which patients are directed, arrival time, demographic data, triage color, start and end time of treatment, decision time and decision type.

The average number of patient arrivals per day is 729. According to Figure 2, concerning the arrival pattern, the ED is busy between 9 a.m. and 11 p.m. and average patient inter arrival time is 1 hour and 45 minutes. Between 11 p.m. and 7 a.m. the ED is idle and the average patient inter arrival time is 11 hour and 64 minutes.

The distribution probabilities of patients at the treatment locations are as follows: $1.5 \%$ of patients enter to the red patient area, $39 \%$ the yellow patient area, $24 \%$ the green patient area and the remained $35 \%$ the injection room. Although the HBYS database provides information on the ED from patient data, some of the data used in the simulation model are not obtained directly from the automation system. Hence, they were collected from onsite observation and the opinion of professional staff.

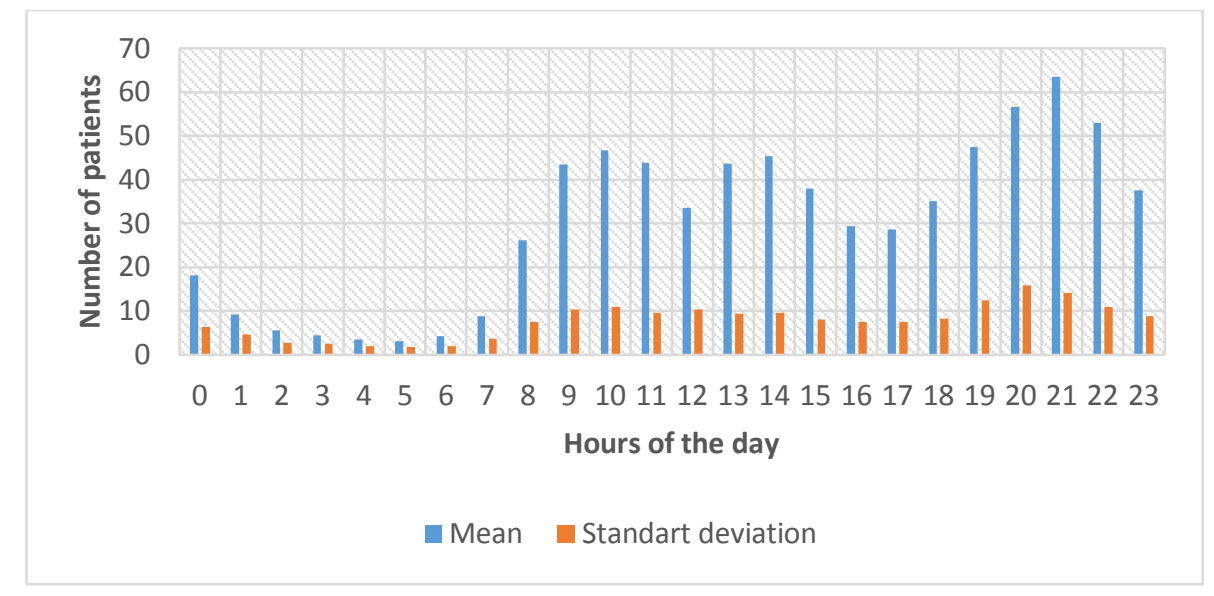

Figure 2: Patient arrival pattern

\subsection{Model implementation}

Based on gathered data, the ED simulation model is built using a multimethod simulation software called AnyLogic Professional 6.4.1 (http://www.anylogic.com). ED systems consist of numerous stochastic factors such as arrivals and service processes. These complicated systems might be difficult to model using analytical models (e.g. queuing theory, mathematical programming). Moreover, ED decision makers can easily gain an understanding of and make changes to a 
simulation model through a user-friendly interface and animation [20]. Therefore, in this study, a discrete event simulation approach was used to model the ED system and monitor its changes in a disaster scenario. To design the ED DES model, some assumptions and simplifications were made. Firstly, walk-in and ambulance patient arrivals were combined into patient arrivals. Secondly, the ED has no appointment system. Thirdly, the registration area and the queues have an infinite capacity. Fourthly, the service times are fitted to a triangular distribution except for services in the injection room (Table 1). Figure 3 shows that it follows a Weibull distribution. The Weibull distribution is a continuous distribution bounded on its lower side. It has shape and scale parameters. In particular, the Weibull distribution is used to represent health related issues, reliability and so on [24]. Suitable distribution functions fitting the data were selected using the Arena Input Analyzer. The Weibull distribution was selected for the service time in the injection room, given that it has a smaller square error (0.000225) than other distribution function alternatives proposed by the software. Moreover, the distribution for service time in the injection room is statistically tested using Chi Square and Kolmogorov Smirnov goodness of fit tests.

\begin{tabular}{|c|c|c|}
\hline Room name & Distribution name and parameters & Number of resources \\
\hline Green & Triangular $(15,18,20)$ & 4 nurses \\
\hline Yellow & Triangular $(35,45,50)$ & 4 nurses \\
\hline Red & Triangular $(60,100,140)$ & 2 nurses \\
\hline Injection & $1+$ Weibull $(20.4,1.19)$ & 2 nurses \\
\hline
\end{tabular}

Table 1: Service time distributions

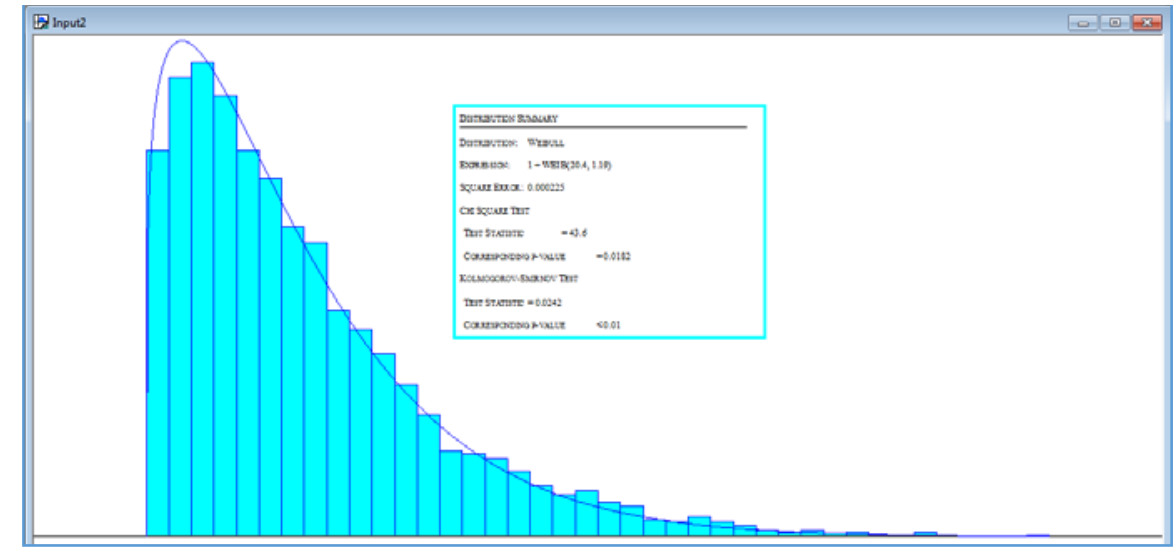

Figure 3: The Weibull distribution function fitted to the service time in the injection room of the observed ED

Thereafter, the ED simulation model is verified and validated. Verification is carried out by a computer program that constructed the simulation model. The computer program determines whether the model works properly as a 
presentation of a real world system. Validation aims to determine whether the model is a picture of a real world system. This is complete when the model logic and inputs are constructed accurately on the computer. Various approaches are used in the literature for verifying and validating the simulation models. These are correctness of data, animation of the model and correctness of operations and outputs [9]. The computer model is checked whether it flows correctly. Then the model is run in a pilot research, and the ED executive of the observed real ED system was asked to evaluate the simulation model. The evaluation was positive and accompanied by an explanation that the model simulates quiete accurately the process flow in the real ED system.

The simulation model has one source that creates patients according to the rates in Figure 2. The source element of the model built using AnyLogic's Enterprise Library, which is a library for building process-centric discrete event simulations, is assigned a category of patients. Figure 4 shows a snapshot of the ED simulation model.

The inputs from the ED simulation model include patient arrival rates (shown in Figure 2) and treatment times in each ED area. Since the interarrival times (IATs) are very random and no distribution with a good fit was found, an indirect method is followed in modelling patient arrivals [18]. Some performance metrics (in other words KPIs) were used, such as average LOS, average throughput (number of patients discharged from the ED), utilization of personnel and utilization of locations.

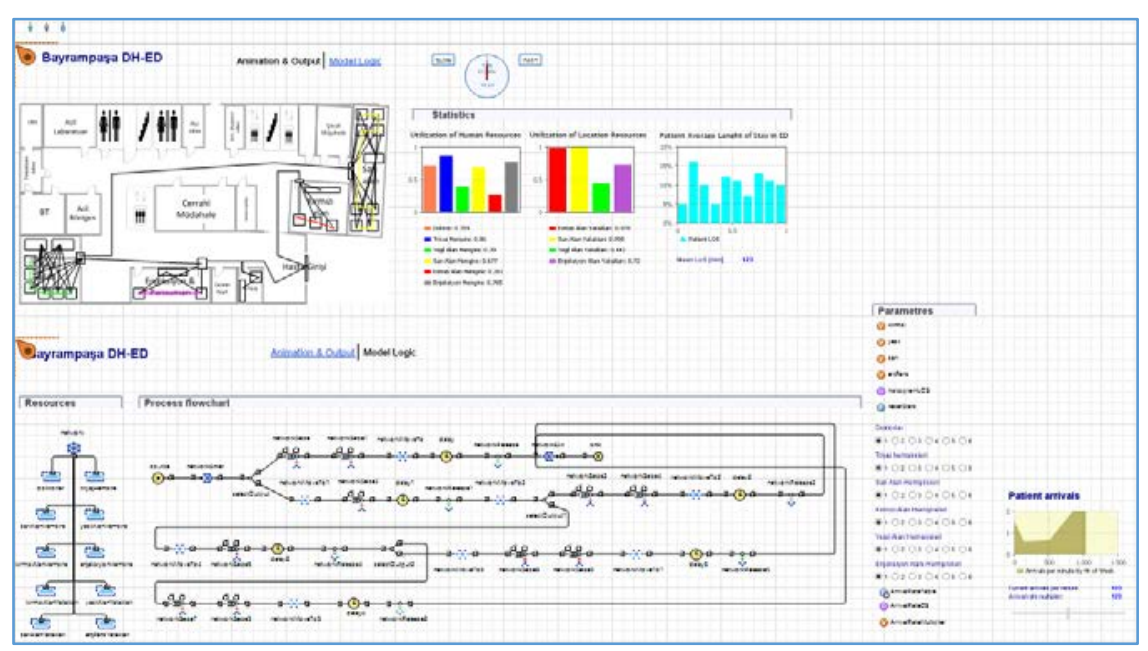

Figure 4: A screenshot showing the ED simulation model

\section{Model results and patient surge scenario}

The values of the average patient LOS and resource utilizations during normal times are obtained after the model was run as a as-is scenario. According to this current scenario, the following can be concluded: (1) the patient LOS is on 
average 168 minutes; (2) the busiest human resources of the ED are nurses who are responsible for the injection room with a utilization rate of $77 \%$, and the idlest of them are nurses in the red patient area with a utilization rate of $23 \%$; and (3) as a result of these utilization rates of human resources, the busiest location is the injection room with a utilization rate of $76 \%$ whereas the idlest is the red patient area with a utilization rate of $19 \%$.

In addition to the analysis of normal time operations in the ED using the simulation model, patient surge was carried out based on disaster time scenarios. Xiao et al. [25] defines the patient surge as the distortion in the ratio of patient numbers to the amount of ED resources, leading to an inadequate ability in facing demand.

As emphasized above, EDs face an increase in patient demand during disasters. According to the results of a study by Dursun et al. [7] for the earthquake case of an ED in Van, Turkey, patient arrivals increase 30\% with respect to the normal state on the first day of the disaster. The total number of arrivals comprises $94 \%$ of disaster-victim patient arrivals and $6 \%$ of nondisaster-victim patient arrivals. Therefore, additional scenarios for different arrival rates and patient type percentage as [17] were devised. Table 2 shows proposed disaster scenarios according to different arrivals.

\begin{tabular}{|c|c|c|c|c|}
\hline \multirow{2}{*}{ Model } & \multirow{2}{*}{ Arrival rate } & \multicolumn{3}{|c|}{ Patient type percentage } \\
\cline { 3 - 5 } & & Red & Yellow & Green and Injection \\
\hline As-is scenario & $729 \mathrm{pts} /$ day & $1.5 \%$ & $24 \%$ & $74.5 \%$ \\
\hline Scenario 1 & $948 \mathrm{pts} /$ day & $10 \%$ & $30 \%$ & $60 \%$ \\
\hline Scenario 2 & $765 \mathrm{pts} /$ day & $5 \%$ & $30 \%$ & $65 \%$ \\
\hline Scenario 3 & $948 \mathrm{pts} /$ day & $20 \%$ & $40 \%$ & $40 \%$ \\
\hline
\end{tabular}

Table 2: Scenario settings

The as-is scenario uses an arrival rate of 729 patients per day with percentages of $1.5 \%, 24 \%$ and $74.5 \%$ for red, yellow and green \& injection room patients, respectively. The remaining three scenarios include both an increase in the arrival rate and a change in the percentage of patient type. Scenario 1 represents an alert for disaster time based on a percentage of $10 \%$ for red patients, $30 \%$ for yellow patients and $60 \%$ for green and injection room patients with an arrival rate of 948 patients per day. Scenario 2 decreases the percentage of red patients from $10 \%$ to $5 \%$ and increases the percentage of green \& injection room patients from $60 \%$ to $65 \%$, while maintaining the yellow patient percentage for the first scenario that is based on an arrival rat of 765 patients per day. Scenario 3 imposes conditions representing a major disaster case for the ED. It assumes a percentage of $20 \%$ for red patients and $40 \%$ for yellow and green \& injection room patients based on an arrival rate of 948 patients per day. This scenario was developed to take into account the expected increase in the number of severely injured patients due to a major disaster compared to normal times. The complete results from running the ED simulation model for these scenarios are shown in Table 3. 


\begin{tabular}{|c|c|c|c|c|c|c|c|c|c|c|c|}
\hline & \multirow{3}{*}{ LOS $(\min )$} & \multicolumn{10}{|c|}{ Utilization rates (\%) } \\
\hline & & \multicolumn{6}{|c|}{ Human resources (HR) } & \multicolumn{4}{|c|}{ Location resources (LR) } \\
\hline & & HR1 & HR2 & HR3 & HR4 & HR5 & HR6 & LR1 & LR2 & LR3 & LR4 \\
\hline As-is scenario & 168.315 & 35.2 & 72.8 & 71.0 & 74.7 & 22.6 & 76.4 & 17.8 & 36.4 & 67.4 & 75.9 \\
\hline Scenario 1 & 203.213 & 34.0 & 72.2 & 50.6 & 78.0 & 86.3 & 74.0 & 80.5 & 38.1 & 36.2 & 73.7 \\
\hline Scenario 2 & 178.482 & 36.1 & 73.7 & 61.1 & 80.6 & 63.8 & 77.8 & 62.4 & 39.3 & 49.9 & 77.5 \\
\hline Scenario 3 & 364.326 & 33.1 & 68.8 & 51.6 & 79.4 & 98.4 & 75.2 & 98.4 & 38.7 & 38.1 & 78.0 \\
\hline
\end{tabular}

Note: HR1: Doctors, HR2: Triage nurses, HR3: Green area nurses, HR4: Yellow area nurses, HR5: Red area nurses, HR6:Injection room nurses, LR1: Red patient area, LR2: Yellow patient area, LR3: Green patient area, LR4: Injection room

Table 3: Complete results of the disaster-based scenarios

According to Scenario 1 and Scenario 3 that test ED behavior in a possible disaster, total patient LOS increases by $20.73 \%$ (from 168,315 to 203,213) and $116.45 \%$ (from 168,315 to 364,326 ) as against the as-is scenario. A remarkable result is the utilization rate of red area nurses and the red patient area. The results of Scenario 1 indicate that red area nurses and the red patient area incur a $281.86 \%$ and $352.25 \%$ increase to their utilizations, respectively. In Scenario 3, the utilization rates have higher scores than the Scenario 1. While the utilization of red area nurses leads to an increase of $335.40 \%$, the red patient area has a percentage of $452.81 \%$ (see Figure 5). This means that in times of a possible disaster, when the percentage of red patient arrivals exceeds $20 \%$ of the total patient arrivals, the number of red area nurses and the space of the red patient area will be insufficient for the ED. In such cases, management needs additional resources to respond to the patient surge.

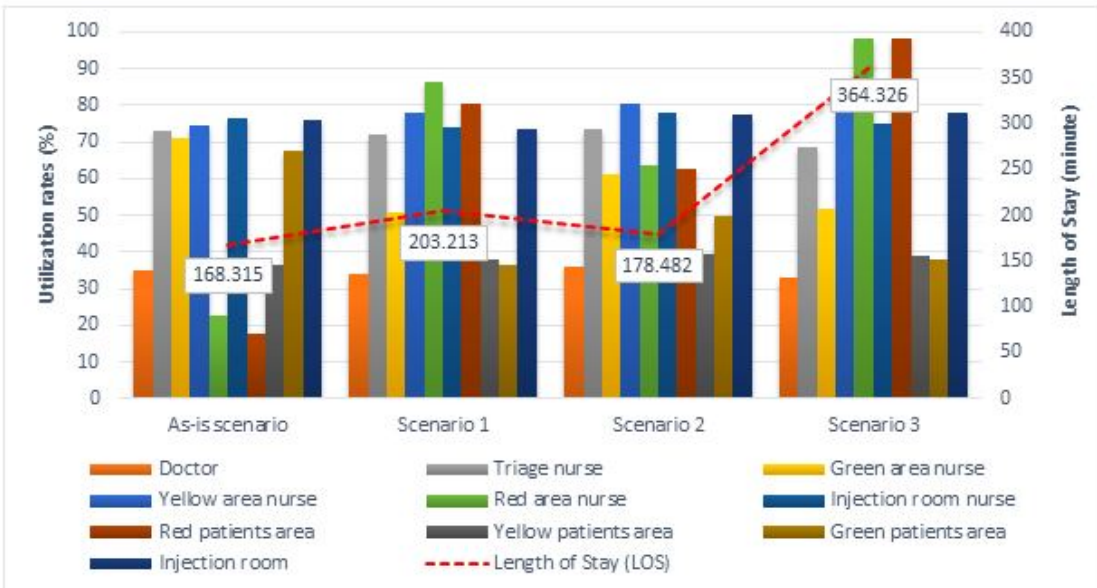

Figure 5: Comparison of the scenarios under four arrival configurations

The major disaster case scenario results in a shortage of two important types of resources. Firstly, available nurses in the red patient area will not be adequate to handle the large number of expected victims. To overcome problems associated with a shortage of resources, the suggestion is for the ED to outsource nurses. This staff should be available as soon as possible in the case of 
a major disaster event. Hence, this suggestion can be performed by means of dispatching them from some of the inpatient departments, which are less burdened during disasters. The injection room nurses who are the busiest human resources in the ED, according to the normal time scenario, can be utilized in order to handle shortages in case of a major disaster. On the other hand, ED management will be faced with a bed shortage. They are advised to set up mobile hospitals necessary for recovery. Under the extreme case (a major disaster) of an arrival rate of 948 patients per day, the average LOS is 364.326 minutes. This implies that improving ED performance is hardly required to reduce the LOS.

As an improvement to the methodology, a different distribution function was tested for the service time of the injection room. The distribution fitting software proposes a number of distribution functions fitted to our data. However, they should be tested for goodness of fit. The Weibull function was used for the service process in the injection room in our model, given that it has the least square error. We then decided to propose an alternative distribution function in the form of $1+$ Gamma $(14.3,1.34)$ with a square error of 0.000230 . The abovementioned Gamma distribution function is presented in Figure 6.

The as-is scenario was run with fifty replications that considered the service time of the injection room fitted to Gamma distribution. It compared patient LOS values with respect to service time of the injection room using both Weibull and Gamma functions subject to a hypothesis test for a mean (T-test) as in Table 4. It shows that p-value is less than 0.05 . The comparison verifies that the patient LOS derived from a comparison between the distribution functions of the injection room service process (Weibull and Gamma) is significantly different.

The conclusion is that the Weibull distribution function used in the service process of the injection room fits the as-is scenario better than Gamma distribution function.

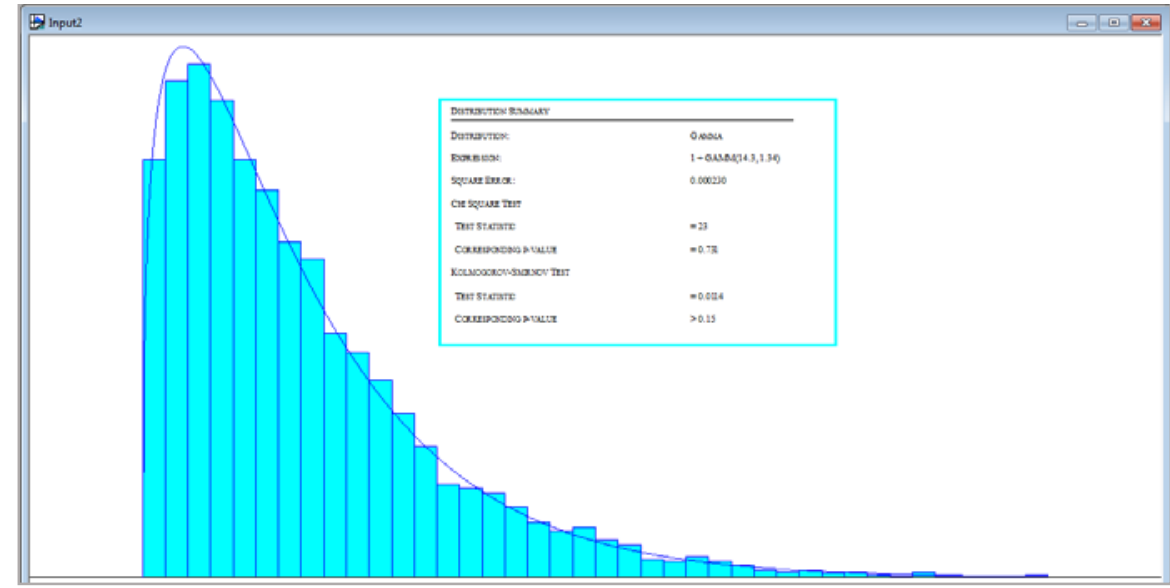

Figure 6: Gamma distribution function fitted to service time in injection room of the observed $E D$ 


\begin{tabular}{|c|c|c|c|}
\hline KPI & \multicolumn{2}{|c|}{$\begin{array}{c}\text { Model output (Distribution function of injection room service } \\
\text { time in minutes) }\end{array}$} & $\begin{array}{c}\text { Significance } \\
\text { (p-value) }\end{array}$ \\
\hline $\begin{array}{c}\text { Patient } \\
\text { LOS }\end{array}$ & $1+$ Weibull (20.4, 1.19) & $1+$ Gamma (14.3, 1.34) & \multirow{2}{*}{$0.000<0.05$} \\
\cline { 2 - 3 } & 168.315 & 188.165 & \\
\hline
\end{tabular}

Table 4: Comparison of different distribution functions for service time in the injection room

\section{Conclusion}

In this study, a DES study of an ED in a public hospital located in an earthquake zone in Istanbul, Turkey, was presented in order to analyze both normal operational time of an ED and that of a disaster time scenario by considering increased disaster-victim patient arrivals. The DES, which has a long history in simulation methodologies, has been applied for ED operations in this study given that it has the ability to test normal and disaster times of the related scenarios. Data was collected on patient arrival patterns and service times in each treatment area of the ED. Using a distribution-fitting tool, the related data was fitted to suitable theoretical distribution functions. The patient flow of the observed hospital ED was conceptually introduced and a DES model was created using commercial simulation software. By constructing a simulation model, four scenarios (the As-Is Scenario and Scenarios 1, 2 and 3) were created to represent the changes to KPIs resulting from a disaster-related patient surge.

The study is different from other studies in a number of aspects. First, it presents a DES model of the ED enabling generation of various scenarios that depend on patient arrival variability based on a previous earthquake case. The model generates outputs for several KPIs not taken into consideration by previous studies in available literature. Second, it runs and validates the model by changing the distribution function in the service times of treatment areas in order to observe which distribution function fits the model better. Finally, it represents a real-world application study in a public hospital ED. The simulation model performed in this ED is a first attempt to gain an insight into disaster preparedness.

The DES model results show that an ED is affected by patient surges in disaster scenarios. In times of disaster, when the percentage of red patient arrivals exceeds $20 \%$ of total patient arrivals, the number of red area nurses and the space of the red patient area will be insufficient for the emergency department. Consequently, the observed ED cannot adequately meet the patient surge using current human and physical resources. Therefore, the recommendation is that medical staff is temporarily dispatched from other EDs in order to respond to increased demand, or the hiring of external nurses for a time, which would be useful in reducing the average LOS and survival rate of disaster-victims. 
Some limitations in this study should be acknowledged. First, no task switching between medical staff (physicians and nurses) is assumed. This is so because doctor or nurse time spent on more than one patient concurrently is practically difficult. However, in real life situations, medical staff may provide service to several patients concurrently, especially in peak hours. The second limitation of the study concerns the scenario design. Four scenarios based essentially on arrival variability for a possible disaster are devised. However, it can include different scenarios by varying the number of additional medical staff and spaces that are required to keep the KPIs the same as in the as-is scenario. Third is the potential limitation of using one hospital ED case. Although the case is involves one specific hospital, this model can be adapted to any other hospital for its disaster preparedness.

To gain a more comprehensive insight into the level of EDs located in the most risky earthquake zone of Istanbul, the model can be observed as a network of multiple ED DES models. On the other hand, disaster readiness guidelines for executives governing public, university and private hospital EDs at a macro level could be of even greater interest because the guidelines would address collaborative work and decision-making mechanisms for this simulation model. These issues can be addressed in future research.

\section{Acknowledgement}

We thank the General Secretary of Istanbul Fatih Association of Public Hospitals and the emergency department's staff for their guidance and help in gathering data.

\section{References}

[1] Alkan, N., Elmas, İ., Karakuş, M. and Akkay, E. (2001). Doğal afetler sirasında karşılaşılan sorunlar: Bir anket çalışması (Problems encountered during natural disasters: A questionnaire study). Turkish Journal of Trauma \& Emergency Surgery, $7(3), 195-200$.

[2] Al-Kattan, I. and Abboud, B. (2009). Disaster recovery plan development for the emergency department-case study. Public Administration and Management, 13(3), 75-99.

[3] Al-Refaie, A., Fouad, R. H. Li, M. H. and Shurrab, M. (2014). Applying simulation and DEA to improve performance of emergency department in a Jordanian hospital. Simulation Modelling Practice and Theory, 41, 59-72. doi:10.1016/j.simpat.2013.11.010.

[4] Borshchev, A. (2013). The Big Book of Simulation Modeling: Multimethod Modeling with AnyLogic 6. AnyLogic North America.

[5] Cao, H. and Huang, S. (2012). Principles of scarce medical resource allocation in natural disaster relief a simulation approach. Medical Decision Making, 32(3), 470476. doi:10.1177/0272989x12437247. 
[6] Choon, O. H., Dali, Z., Beng, P. T. and Magdalene, C. P. Y. (2014). Uncover effective process improvement strategies in emergency department with aid of discrete event simulation. Health Systems, 3(2), 93-104. doi:10.1057/hs.2014.2.

[7] Dursun, R., Görmeli, C. A. and Görmeli, G. (2012). 2011 Van depremi sonrası Van bölgesi Eğitim ve Araştırma Hastanesine başvuran olguların değerlendirilmesi (Evaluation of the patients in Van Training and Research Hospital following the 2011 Van earthquake in Turkey). Turkish Journal of Trauma \& Emergency Surgery, 18(3), 260-264.

[8] Ghanes, K., Jouini, O., Jemai, Z., Wargon, M., Hellmann, R., Thomas, V. and Koole, G. (2014). A comprehensive simulation modeling of an emergency department: A case study for simulation optimization of staffing levels. In: Proceedings of the 2014 Winter Simulation Conference (pp. 1421-1432). IEEE Press.

[9] Gul, M. and Guneri, A. F. (2012). A computer simulation model to reduce patient length of stay and to improve resource utilization rate in an emergency department service system. International Journal of Industrial Engineering, 19(5), 221-231.

[10] Gul, M. and Guneri, A. F. (2015a). A comprehensive review of emergency department simulation applications for normal and disaster conditions. Computers \& Industrial Engineering, 83, 327-344. doi:10.1016/j.cie.2015.02.018.

[11] Gul, M. and Guneri, A. F. (2015b). A discrete event simulation model of an emergency department network for earthquake conditions. In Modeling, Simulation, and Applied Optimization (ICMSAO), 2015 6th International Conference on (pp. 16). IEEE.

[12] Gul, M. and Guneri, A. F. (2015c). Are emergency departments in Istanbul ready for the earthquakes? Past experience and suggestions for future preparedness from employees' viewpoint and the literature. Journal of Homeland Security and Emergency Management, (in press). doi:10.1515/jhsem-2014-0114.

[13] Gunal, M. M. (2012). A guide for building hospital simulation models. Health Systems, 1(1), 17-25. doi:10.1057/hs.2012.8.

[14] Joshi, A. J. and Rys, M. J. (2011). Study on the effect of different arrival patterns on an emergency department's capacity using discrete event simulation. International Journal of Industrial Engineering: Theory, Applications and Practice, 18(1), 40-50.

[15] Jun, J. B., Jacobson, S. H. and Swisher, J. R. (1999). Application of discrete-event simulation in health care clinics: a survey. Journal of the operational research society, 50(2), 109-123. doi:10.1057/palgrave.jors.2600669.

[16] Kadri, F., Chaabane, S. and Tahon, C. (2014). A simulation-based decision support system to prevent and predict strain situations in emergency department systems. Simulation Modelling Practice and Theory, 42, 32-52. doi:10.1016/j.simpat.2013.12.004.

[17] Kang, H., Nembhard, H. B., Rafferty, C. and DeFlitch, C. J. (2014). Patient flow in the emergency department: A classification and analysis of admission process policies. Annals of Emergency Medicine, 64(4), 335-342.e8. doi:10.1016/j.annemergmed.2014.04.011.

[18] Kaushal, A. (2014). Improving emergency department performance using discreteevent and agent-based simulation. MSc Thesis, University of Manitoba, Canada.

[19] Konrad, R., DeSotto, K., Grocela, A., McAuley, P., Wang, J., Lyons, J. and Bruin, M. (2013). Modeling the impact of changing patient flow processes in an emergency department: Insights from a computer simulation study. Operations Research for Health Care, 2(4), 66-74. doi:10.1016/j.orhc.2013.04.001. 
[20] Kuo, Y. H., Rado, O., Lupia, B., Leung, J. M. and Graham, C. A. (2014). Improving the efficiency of a hospital emergency department: A simulation study with indirectly imputed service-time distributions. Flexible Services and Manufacturing Journal. doi:10.1007/s10696-014-9198-7.

[21] Lim, M. E. Nye, T. Bowen, J. M. Hurley, J. Goeree, R. and Tarride, J. E. (2012). Mathematical modeling: The case of emergency department waiting times. International Journal of Technology Assessment in Health Care, 28(02), 93-109. doi:10.1017/s0266462312000013.

[22] Morgareidge, D. Cai, H. and Jia, J. (2014). Performance-driven design with the support of digital tools: Applying discrete event simulation and space syntax on the design of the emergency department. Frontiers of Architectural Research, 3(3), 250264. doi:10.1016/j.foar.2014.04.006.

[23] Patvivatsiri, L. (2006). A simulation model for bioterrorism preparedness in an emergency room. In: Proceedings of the 38th conference on Winter simulation (pp. 501-508). Winter Simulation Conference.

[24] Stat::Fit Version 2-Statistical Fit Software. Geer Mountain Software Corporation, 2001.

[25] Xiao, N., Sharman, R., Rao, H. R. and Dutta, S. (2012). A simulation based study for managing hospital emergency department's capacity in extreme events. International Journal of Business Excellence, 5(1), 140-154. doi:10.1504/ijbex.2012.044578. 